\title{
A response-regulator-like activator of antibiotic synthesis from Streptomyces coelicolor A3(2) with an amino-terminal domain that lacks a phosphorylation pocket
}

\author{
Ellen P. Guthrie, Christine S. Flaxman, Janet White, David A. Hodgson, Mervyn J. Bibb \\ and Keith F. Chater
}

Microbiology (1998), 144, 727-738

p. 732, Fig. 3 legend

The base change in the cloned Pwb-6 mutant DNA is shown at nucleotide position 62 .

should read

The base change in the cloned Pwb-9 mutant DNA is shown at nucleotide position 62 .

p. 735 , first paragraph

Analysis of the sequence upstream of this from Pwb-6 DNA revealed a base change in the -35 region of the redZ promoter (sequences upstream of the coding regions of redZ in Pwb-9 and Pwb-16 DNA have not yet been determined). This change caused a closer resemblance to the likely consensus -35 sequence for promoters presumed to be recognized by the major vegetative form of RNA polymerase holoenzyme:

TCTCCA Pwb ${ }^{+}$

TTTCCA Pwb-6

TTGACA -35 consensus

should read

Analysis of the sequence upstream of this from Pwb-9 DNA revealed a base change in the -35 region of the redZ promoter (sequences upstream of the coding regions of redZ in Pwb-6 and Pwb-16 DNA have not yet been determined). This change caused a closer resemblance to the likely consensus -35 sequence for promoters presumed to be recognized by the major vegetative form of RNA polymerase holoenzyme:

TCTCCA Pwb ${ }^{+}$

TTTCCA Pwb-9

TTGACA -35 consensus 